\title{
IMPACTO DA NÃO-ADESÃO DO RECEPTOR NOS DESFECHOS EM LONGO PRAZO DE TRANSPLANTE. REVISÃO DA LITERATURA E EXPERIÊNCIA DA UNIVERSIDADE DE MINNESOTA
}

\author{
Impact of Recipient Non-adherence on Long-Term Transplant Outcome. \\ Literature review and experience of the University of Minnesota.
}

Arthur J. Matas, MD

\section{RESUMO}

Nas últimas duas décadas houve um aumento significativo na sobrevida do paciente e do enxerto no pós-transplante. Entretanto, não houve melhora paralela nos resultados em longo prazo. Uma causa potencialmente remediável da perda tardia de enxerto é a não-adesão ao tratamento imunossupressor. Não é possível quantificar com precisão a percentagem de perda de enxerto atual devida à não-adesão, porque existem muitos aspectos envolvidos (ex., ingestão de medicação imunossupressora e outras, além do acompanhamento clínico). Ainda não há uma métrica uniforme de avaliação e existe uma falta de definição clara do que seja não-adesão. Vários estudos em transplante renal, cardíaco e hepático mostraram associação entre não-adesão e maior incidência de episódios de rejeição aguda, crônica, perda tardia de enxerto e necessidade de re-transplantes. Esses resultados têm conseqüências econômicas significativas para o Sistema de Saúde. Deve-se considerar alguns fatores para a decisão de se fazer um re-transplante em um paciente com perda de enxerto por não-adesão. Em pacientes transplantados de coração ou fígado, a recusa ao re-transplante levará ao óbito mas, para pacientes com insuficiência renal, a diálise é uma opção de tratamento. Nossa conduta é considerar tais pacientes para um segundo transplante, se eles demonstrarem que são aderentes ao regime de diálise por um mínimo de seis meses. Não será feito terceiro transplante em pacientes que tenham perdido os dois transplantes prévios por não-adesão.

Descritores: Transplante, Rejeição de Enxerto, Imunossupressão, Terapia.

Institution

Department of Surgery, University of Minnesota, 328, 420 Delaware Street SE, Minneapolis, MN 55455, USA.

\section{Address:}

Arthur J. Matas, MD

Department of Surgery, University of Minnesota

328, 420 Delaware Street SE, Minneapolis, MN 55455, USA.

E-mail: matas001@umn.edu

Com apoio financeiro da NIH \#DK13083

Arthur J.Matas recebeu apoio financeiro da Janssen-Cilag Farmacêutica.

\section{INTRODUÇÃO}

Nas últimas quatro décadas, o índice de sobrevida em curto prazo (um ano) do enxerto renal melhorou consideravelmente, mas com pequena melhora concomitante na sobrevida do enxerto no longo prazo. Nos Estados Unidos, o Registro Científico de Resultados de Transplantes (SRTR) relata um índice de sobrevida dos enxertos em dez anos em transplantes de órgãos sólidos de cerca de $50 \%$ ou menos (comunicação pessoal). Para o transplante renal, o índice de sobrevida do enxerto em dez anos contabilizado por óbito com doador vivo é de apenas $64 \%$ e com doador falecido, $52 \%$.

$\mathrm{Na}$ Universidade de Minnesota estudamos as causas da perda de enxerto ao longo do tempo em pacientes receptores de transplante renal. ${ }^{1,2}$ Conseguimos demonstrar que nos anos 80 (comparativamente aos 70), o índice de perda de enxerto por rejeição aguda foi significantemente menor, porém, não foi demonstrada qualquer alteração no índice de perda de enxerto por rejeição crônica (nefropatia crônica do enxerto [NCE]). ${ }^{1}$ Para transplantes renais realizados nos anos 90 em nossa instituição, a rejeição crônica persistiu como causa importante da perda de enxerto pós-transplante. Quando subdividimos os receptores com perda de enxerto por motivo de perda, observamos que a trombose por questões técnicas e o óbito com enxerto funcionante foram as causas 
mais comuns no primeiro ano pós-transplante, enquanto o óbito com enxerto funcionante e rejeição crônica ( $\mathrm{NCE}$ ) foram as causas mais comuns após o primeiro ano. ${ }^{2}$ Contudo, a clara falta de adesão (que definimos como admissão explícita por parte dos receptores que cessaram completamente ou diminuíram a ingestão da medicação) associou-se com perda do enxerto em todos os intervalos de tempo que estudamos. Por exemplo, no intervalo de um a cinco anos póstransplante, cerca de $11 \%$ das perdas de enxerto foram devidas à não-adesão, e, após cinco anos de $9 \%$.

Entretanto, é provável que o impacto da não-adesão sobre a perda de enxertos seja sub-relatada. Em uma publicação inicial, Gaston et al. estudaram a perda tardia do enxerto em 1085 receptores com sobrevida de enxerto por seis meses. ${ }^{3}$ Dos 1084 receptores, 184 subseqüentemente perderam seus enxertos. Aqueles autores observaram que quatro dessas perdas ( $2 \%$ ) estavam declaradamente relacionadas com a não-adesão ao tratamento. Os autores fizeram então uma revisão detalhada da ficha dos 184 receptores com perda do enxerto, e observaram que era possível documentar uma nãoadesão significante em 48 dos 184. Após a revisão, a não-adesão foi documentada como a segunda causa mais comum de perda do enxerto na população de pacientes.

\section{QUESTÕES REFERENTES A ESTUDOS DE NÃO- ADESÃO}

Os achados desses autores auxiliam na elucidação de um dos problemas principais na tentativa de compreender o impacto da não-adesão nos resultados do enxerto - isto é, a não-adesão é mal definida nos registros médicos. Recentemente, Dew \& Dimartini observaram três fatores que limitam o progresso na compreensão da não-adesão pós-transplante: deixar de considerar todos os aspectos da não-adesão, falta de mensurações úteis e poucas tentativas de uma síntese entre estudos. ${ }^{4}$ Esses autores notaram que a maioria dos estudos enfatiza apenas um aspecto da não-adesão do receptor (por exemplo, após transplante renal, deixar de tomar a medicação; após transplante hepático, voltar ao uso de álcool). Porém, a adesão ao regime terapêutico pós-transplante compreende múltiplos componentes, que incluem a tomada dos imunossupressores e de outras medicações, comparecimento às consultas agendadas, realização dos exames de sangue, monitoramento dos sinais vitais, manutenção de uma dieta e um nível de atividade física adequados para redução do risco de obesidade e evitar o abuso de álcool, tabagismo ou uso de substâncias ilícitas. Dew et al. observaram que a definição e a documentação desses tipos individuais de não-adesão variaram amplamente entre estudos e entre tipos de não-adesão. ${ }^{5}$ Por exemplo, identificaram que a não tomada das medicações imunossupressoras foi documentada mais freqüentemente do que ausência às consultas agendadas. Eles também demonstraram que o tipo de não-adesão (medido por casos - 100 pessoas/ano) variou amplamente conforme o órgão transplantado. Segundo a literatura de revisão, 36\% dos receptores de rim foram identificados como não aderentes quanto à tomada das medicações imunossupressoras, em comparação com $15 \%$ dos receptores de coração e $7 \%$ dos receptores de fígado. Por outro lado, $47 \%$ dos receptores de coração foram não-aderentes quanto ao monitoramento dos sinais vitais, em comparação com $6 \%$ dos receptores de rim. Também foi relatado dificuldades na tentativa de desenvolver uma perspectiva global da não-adesão e observaram que poucas variáveis psicossociais significativas estavam associadas de forma coerente nos estudos de não-adesão. ${ }^{5}$ Embora talvez se possa sugerir que alguns grupos (por exemplo, adolescentes) têm maior risco de não-adesão, a previsão de não-adesão de um receptor individual é impossível.

Dew et al. demonstraram que a não-adesão quanto à tomada das medicações imunossupressoras tendeu a se associar com a declaração de estado de saúde desfavorável, pouco apoio social e etnia não branca. Da mesma forma, a não-adesão quanto ao acompanhamento de saúde associou-se ao pouco suporte social e etnia não branca. O fator mais importante na previsão do comportamento pós-transplante foi o abuso de substâncias.

Em nossa prática clínica a história relevante de abuso de substâncias só foi observada em poucos receptores. É digno de nota que fatores de risco comumente aceitos para não-adesão, como a idade do receptor, não foram bons preditores.

\section{IMPACTO DA NÃO-ADESÃO NO RESULTADO EM LONGO PRAZO}

Schweitzer et al. observaram inicialmente o impacto da não-adesão no resultado pós-transplante em estudo de 538 receptores de rim, 50 de coração e 13 de fígado. ${ }^{6}$ Em estudo retrospectivo de 260 receptores de rim, observaram que a não-adesão ocorreu em 18\% deles; 91\% dos receptores não aderentes perderam o enxerto ou morreram e em outro estudo prospectivo de 196 receptores de rim observaram que a não-adesão ocorreu em 15\%. Duas importantes observações feitas e corroboradas por outros estudos subseqüentes foram: (1) a não-adesão podia ocorrer muitos anos após o transplante (o que foi observado nos três tipos de transplante estudados coração, fígado e rim); (2) a não-adesão foi, em geral, não-previsível e freqüentemente não tinha uma razão identificável.

Mais tarde, De Geest et al. observaram que 23\% dos receptores de transplante renal entrevistados mais de um ano após o transplante eram não-aderentes à tomada das medicações imunossupressoras. ${ }^{7}$ A não-adesão se associou a um índice mais elevado de episódios agudos de rejeição $(\mathrm{p}=0,003)$ e com um índice significantemente mais baixo de sobrevida do enxerto aos cinco anos $(p=0,03)$.

Recentemente, Takamoto et al. estudaram a adesão em receptores cujo seguro-saúde era fornecido pela Medicare pela determinação do índice de fornecimento de uma nova prescrição (definindo adesão como o novo fornecimento de prescrições). ${ }^{8}$ Eles agruparam os pacientes em quartis, com base na adesão (alta, média, baixa e ruim). Os receptores com alta adesão tiveram um índice de sobrevida do enxerto significantemente mais alto, quando comparado a cada um dos outros grupos).

De Geest et al, utilizando chips de computador nas tampas das medicações imunossupressoras, estudaram o impacto da nãoadesão em receptores de coração. ${ }^{9}$ Agruparam os receptores no que denominaram "excelente adesão" (84\%), "não aderentes mínimos sub-clínicos" (7\%) e "não aderentes moderados sub-clínicos" (9\%). A não-adesão se associou com aumento significante no índice de rejeição aguda tardia $(\mathrm{p}=0,01)$.

Em um seguimento de cinco anos, Dobbels et al. observaram que os grupos de não aderentes tinham um risco 4,9 vezes maior de rejeição aguda tardia, risco 5,4 vezes maior de necessidade de retransplante e risco uma vez maior de arteriopatia coronariana no transplante..$^{10}$ Em outro estudo de receptores de coração, Dew et al. observaram que os receptores não aderentes tiveram um índice 4,2 vezes maior de rejeição aguda e risco 6,9 vezes maior de doença arterial coronária no transplante. ${ }^{11}$ 
Em adolescentes receptores de fígado, Berquist et al. observaram: 1) que os receptores não aderentes tiveram um risco significantemente maior de rejeição aguda tardia $(\mathrm{p}<0,001)$ e de rejeição aguda tardia sem terem apresentado um episódio precoce de rejeição aguda $(\mathrm{p}<0,001) ; 2)$ necessidade de retransplante devido a rejeição crônica $(\mathrm{p}<0,006)$ e de óbito por rejeição crônica $(\mathrm{p}<0,05) .{ }^{12}$

O'Carroll et al. observaram que receptores de fígado que faltaram ou cancelaram $>25 \%$ de suas consultas após o transplante tinham maior probabilidade de óbito no acompanhamento tardio (em comparação com receptores que compareceram às visitas agendadas); semelhantemente, pacientes com rejeição celular tardia ( $>6$ meses), quando comparados aos sem rejeição, tiveram maior probabilidade de óbito no acompanhamento tardio. ${ }^{13}$

Duas metanálises verificaram o impacto da não-adesão no resultado do transplante renal, cada uma delas em períodos de tempo diferentes. Butler et al. em estudos publicados de 1980 a 2001, selecionaram 36 de 325 estudos que cumpriram os seus critérios de inclusão. ${ }^{14}$ Alguns desses 36 estudos eram populacionais e descreviam a prevalência da não-adesão em uma população clínica de receptores com enxertos funcionantes e neles, o índice de não-adesão foi em média $22 \%$ (variando de $12 \%$ a 26\%). Em 86/325 cujos estudos eram de coorte, isto é, incluíram uma população de receptores em um período de tempo definido, o índice de não-adesão foi em média $15 \%$ (variando entre $5 \%$ e $20 \%$ ). Nos estudos de coorte, $36 \%$ das perdas de enxerto se associaram à não-adesão. Na metanálise geral dos 36 estudos, Butler et al. observaram que as tendências à perda do enxerto foram sete vezes maiores em receptores não aderentes (em comparação com aderentes) [p<0,001].

Na segunda metanálise, que envolveu literatura de 1988 até 2004, Denhaerynck et al. observaram a prevalência de não-adesão em $28 \%$ e concluíram que a não-adesão contribuiu para $20 \%$ dos episódios de rejeição aguda tardia e $16 \%$ das perdas de enxerto. ${ }^{15}$

\section{EXPERIÊNCIA DA UNIVERSIDADE DE MINNESOTA}

Em nossa universidade, utilizamos chips de computadores nos frascos de medicações imunossupressoras de nossos receptores com a finalidade de medir a adesão ao tratamento. Os chips registram se o frasco foi aberto por tempo suficiente para retirar o comprimido. Com este método, presumimos que se um receptor abriu o frasco de medicação por aquele intervalo de tempo, também terá tomado a medicação.

Originalmente, monitoramos os frascos para os comprimidos de azatioprina (tomada uma vez ao dia) e posteriormente, monitoramos os frascos dos comprimidos de micofenolato mofetil ou de sirolimo. Dessa forma, presume-se também que um receptor que abre um de diversos frascos de comprimidos, provavelmente abrirá os demais e também tomará as demais medicações.

Em nosso estudo inicial, agrupamos 180 receptores em quartis com base em sua adesão à tomada de azatioprina. ${ }^{16}$ É digno de nota que o grupo com adesão mais baixa teve maior índice de rejeição aguda $(\mathrm{p}=0,0006)$ e maior índice de perda do enxerto $(\mathrm{p}=0,002)$. Os receptores cuja adesão caiu progressivamente nos primeiros três meses de seguimento tiveram o pior resultado pós-transplante $(\mathrm{p}=0,001)$. Os receptores no quartil de melhor adesão não tiveram rejeição aguda, nem perda de enxerto durante os dois a quatro anos subseqüentes.

Até o momento, seguimos 180 receptores por um tempo médio de 8,7 anos, e uma importante observação é que cada um dos quatro grupos de quartis teve adesão decrescente com o passar do tempo.
Talvez mais importante, foi o fato que os receptores com diminuição de adesão nos primeiros três meses pós-transplante tiveram um aumento significante de índice de rejeição aguda $(\mathrm{p}=0,001)$, rejeição aguda tardia $(p=0,03)$, perda do enxerto $(p=0,001)$ e óbito $(p=0,04)$. Assim, precocemente após o transplante, pode-se definir que um grupo de receptores tem risco significantemente aumentado de perda do enxerto, devido a não-adesão ao tratamento. Atualmente, estamos estudando se a intervenção nesse grupo de alto risco levará a um melhor resultado.

\section{RETRANSPLANTE PARA RECEPTORES COM FALHA DO ENXERTO POR NÃO-ADESÃO}

Uma importante consideração é sobre realizar ou não retransplante para receptores com falha do enxerto por não-adesão. Para receptores de coração, pulmão, ou fígado, a não realização do retransplante levará o paciente à morte, contrariamente aos receptores de rim, onde existe a terapia alternativa da diálise.

Por isso, seria possível recusar o retransplante para receptores de rim não-aderentes, e condená-los a passar o resto de suas vidas submetendo-se à diálise. Entretanto, sabemos que a sobrevida do paciente e a qualidade de vida são significantemente melhores com retransplante (em comparação com a diálise).

Na Universidade de Minnesota, nossa conduta é considerar o retransplante para receptores selecionados com perda de enxerto por não-adesão. Fazemos uma extensa reavaliação do candidato a retransplante, inclusive com uma determinação das razões para a não-adesão após o primeiro transplante, e tentamos desenvolver um plano para prevenção da não-adesão após o retransplante. Essa reavaliação inclui uma avaliação psicossocial completa. Em seguida, insistimos em um mínimo de seis meses de tratamento dialítico para verificar se o candidato demonstra adesão a todos os cuidados da diálise, inclusive uso correto das medicações e comparecimento a todas as consultas agendadas. Esclarecemos ao candidato que, se o segundo enxerto for perdido por não-adesão, um terceiro transplante em nossa instituição não será considerado. Quando há envolvimento de doador vivo, apresentamos nossa preocupação com a não-adesão do doador, asseguramo-nos que o doador compreenda as implicações da não-adesão do receptor e solicitamos ao doador que se envolva em um contrato informal com o candidato, pedindo adesão à tomada das medicações imunossupressoras e manutenção de todos os agendamentos de acompanhamento como condição da doação. Apenas se todas as fases desse procedimento forem totalmente cumpridas, consideramos o paciente para retransplante. Até aqui, nossa experiência, não obstante os pequenos números sugere que se os candidatos são selecionados cuidadosamente, um retransplante pode ser bem sucedido, mesmo após a perda do primeiro enxerto por não-adesão. ${ }^{17}$

\section{O FUTURO}

Pode-se concluir que a não-adesão à tomada das medicações imunossupressoras tem conseqüências muito graves, inclusive causando aumento dos índices de rejeição aguda, rejeição aguda tardia, rejeição crônica, com perda do enxerto, retransplantes e óbito. Contudo, continua difícil definir não-adesão e realizar estudos de prevenção e por isso, é necessário que sejam realizados mais estudos para padronizar as definições e determinar como melhor prever e prevenir a não-adesão ao tratamento imunossupressor. 


\section{ABSTRACT}

In the last 2 decades, there has been a significant after-transplantation improvement of patient and graft survival in short-term. But there has not been a parallel improvement in the long-term outcome. A potentially remediable cause for the late graft loss is the non-adherence. It is not possible to precisely quantify if the percentage of graft loss is actually due to non-adherence. Quantifying the graft loss to the non-adherence is not possible, since there are many aspects in the post-transplant adherence (e.g., intake of immunosuppressive and other medications, attending clinic appointments), we do not have a reliable means to measure, and there is a lack of a clear definition on what is non-adherence (e.g., how much medication and/or how many clinic appointments must a recipient miss to be classified as non-adherent?). Several studies in kidney, heart, and liver transplant recipients have shown a clear association between non-adherence and an increased incidence of acute rejection episodes, chronic rejection (chronic allograft nephropathy, or coronary artery disease), late graft loss, and need for re-transplantation. Such outcomes have significant economic consequences for the Healthcare System. Some factors must be considered, in order to decide whether or not to perform the re-transplantation in patients with graft loss due to non-adherence. For heart and liver patients who refuse the re-transplantation, the consequence is death; but for patients with kidney failure, dialysis provides a treatment option. Our policy is to consider such patients for a second transplant if they can show their adherence to the dialysis regimen for a minimum of 6 months period. We will not perform a third kidney transplant in a patient who lost his previous two ones due to non-adherence.

Keywords: Transplantation, Graft Rejection, Immunosuppression, Therapy.

\section{REFERÊNCIAS}

1. Schweitzer EJ, Matas AJ, Gillingham KJ, et al. Causes of renal allograft loss. Progress in the 1980s, challenges for the 1990s. Ann Surg. 1991;214(6):679-88.

2. Matas AJ, Humar A, Gillingham KJ, et al. Five preventable causes of kidney graft loss in the 1990s: a single-center analysis. Kidney Int. 2002;62(2):704-14.

3. Gaston RS, Hudson SL, Ward M, et al. Late renal allograft loss: noncompliance masquerading as chronic rejection. Transplant Proc. 1999;31(4A):21S-23S.

4. Dew MA, Dimartini AF. The incidence of non-adherence after organ transplant: ensuring that our efforts at counting really count. Liver Transpl. 2006;12(12):1736-40.

5. Dew MA, DiMartini AF, De Vito Dabbs A, et al. Rates and risk factors for non-adherence to the medical regimen after adult solid organ transplantation. Transplantation. 2007;83(7):858-73.

6. Schweizer RT, Rovelli M, Palmeri D, et al. Noncompliance in organ transplant recipients. Transplantation. 1990;49(2):374-7.

7. De Geest S, Borgermans L, Gemoets H, et al. Incidence, determinants, and consequences of subclinical noncompliance with immunosuppressive therapy in renal transplant recipients. Transplantation. 1995;59(3):340-7.

8. Takemoto SK, Pinsky BW, Schnitzler MA, et al. A retrospective analysis of immunosuppression compliance, dose reduction and discontinuation in kidney transplant recipients. Amer J Transplant. 2007;7(12):2704-11.

9. De Geest S, Abraham I, Moons P, et al. Late acute rejection and subclinical noncompliance with cyclosporine therapy in heart transplant recipients. J Heart Lung Transplant. 1998;17:854.
10. Dobbels F, De Geese S, van Cleemput J, et al. Effect of late medication noncompliance on outcome after heart transplantation: a 5-year follow-up. J Heart Lung Transplant. 2004;23(11):1245-51.

11. Dew MA, Kormos RL, Roth LH, et al. Early post-transplant medical compliance and mental health predict physical morbidity and mortality one to three years after heart transplantation. J Heart Lung Transplant. 1999;18(6):549-62.

12. Berquist RK, Berquist WE, Esquivel CO, et al. Adolescent non-adherence: prevalence and consequences in liver transplant recipients. Pediatr Transplant. 2006;10(3):304-10.

13. O'Carroll RE, McGregor LM, Swanson V, et al. Adherence to medication after liver transplantation in Scotland: a pilot study. Liver Transpl. 2006;12(12):1862-8.

14. Butler JA, Roderick P, Mullee M, et al. Frequency and impact of non-adherence to immunosuppressants after renal transplantation: a systematic review. Transplantation. 2004;77(5):769-76.

15. Denhaerynck K, Dobbels F, Cleemput I, et al. Prevalence, consequences, and determinants of non-adherence in adult renal transplant patients: a literature review. Transpl Int. 2005;18(10):1121-33.

16. Nevins TE, Kruse L, Skeans MA, et al. The natural history of azathioprine compliance after renal transplantation. Kidney Int. 2001;60:1565-70.

17. Troppmann C, Benedetti E, Gruessner RW, et al. Retransplantation after renal allograft loss due to noncompliance: indications, outcome, and ethical concerns. Transplantation. 1995;59(4):467-71. 